\title{
Synthesis, Characterization, Antidepressant Activity and Docking Studies of Some Novel Indole Bearing Azetidinone Derivatives
}

\author{
Deweshri Rajendrakumar Kerzare*, Sunil Sugnomal Menghani, Pramod Bhujangrao Khedekar \\ Department of Pharmaceutical Sciences, Computer Aided Drug Design Laboratory, Rashtrasant Tukadoji Maharaj Nagpur University, \\ Amravati Road, Nagpur, Maharashtra, INDIA.
}

\begin{abstract}
Context: In general, indole bearing azetidinone derivatives are exhibiting various biological activities. The evaluation of pharmacological potential of the indole bearing azetidinone derivatives as antidepressant agent has been relatively less explored. To get insight of the intermolecular interactions, the molecular docking studies are performed at active site of MAO-A enzyme. Aim: In this study, an attempt has been made to generate new molecular template by linking two pharmacophores (indole and azetidinone), which are likely to exhibit antidepressant-like action in animal models. Methods: The derivatives was synthesized by conventional reactions and characterized by various spectrometric methods. The derivatives were evaluated for antidepressant activity by using forced swim test. Molecular docking studies of the synthesized derivatives with MAO-A enzyme were carried on Vlife MDS Molecular Modelling software, version 4.3.1. by using k-nearest neighbour genetic algorithm method. Results: All the final structures were assigned on the basis of IR, ${ }^{1} \mathrm{H}$ NMR, mass spectra and elemental analyses. The antidepressant evaluation exhibited final derivatives 26 and 36 as promising molecules with percentage decrease in immobility duration 66.82 and 65.61 respectively. Molecular docking studies are also in agreement with pharmacological evaluation with potent compounds exhibiting dock score -2.8474. Conclusion: It can be concluded that these compounds may have enough potential to be developed as antidepressant agent. It can be further studied for their structure-activity relationship (SAR) studies and developed into potential lead molecules. So our research can make a great impact on those medicinal chemists who work on the development of MAO-A inhibitors.
\end{abstract}

Key words: Indole, Azetidinone, Antidepressant activity, Docking study.

\section{INTRODUCTION}

Depression is a prevalent psychiatric disorder with estimates reaching as high as $21 \%$ of the world population. The World Health Organization (WHO) predicts that it will be the second leading cause of death by the year 2020 due to complications arising from stress and the cardiovascular system. ${ }^{1}$ Although a broad range of antidepressants are available today in the market but a significant proportion of these patients will not respond to treatment or will show only partial response. ${ }^{2}$ The physiological reason for depression have not been completely established, but some facts suggested that depressive condition may be due to lack of noradrenaline and serotonin. The majority of the synthetic drugs used in the treatment of such illness act by affecting the system of biogenic amines of the brain, thus leading to action of a mechanism that is capable of increasing their concentration in the brain. Monoamine oxidases (MAO) are a family of enzymes in the CNS that catalyses the oxidation of or inactivation of biogenic amines. ${ }^{3,4}$ MAO inhibitors act by inhibiting the activity of monoamine oxidase, thus preventing the breakdown of monoamine neurotransmitters (noradrenaline and serotonin) and
Submission Date: 19-05-2017; Revision Date: 06-07-2017; Accepted Date: 05-12-2017

DOI: 10.5530/ijper.52.1.13 Correspondence: Deweshri R Kerzare, Department of Pharmaceutical Sciences, Computer Aided Drug Design Laboratory, Rashtrasant Tukadoji Maharaj Nagpur University, Amravati Road, Nagpur 440 033, Maharashtra, INDIA.

Phone no: 9561315019 Email: kerzarepritee@gmail. com

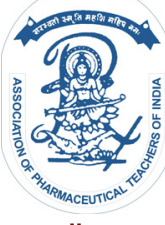

www.ijper.org 
thereby increasing their availability. There are two isoforms of monoamine oxidase, MAO-A and MAO-B. $\mathrm{MAO}-\mathrm{A}$ preferentially deaminates serotonin, melatonin, epinephrine and norepinephrine. MAO-B preferentially deaminates phenethylamine and certain other trace amines. Those agents that can inhibit the above system can produce beneficial change in the condition of the central nervous system. Particularly MAO-A inhibitors have been employed in the treatment of depression, anxiety and mental disorders while MAO-B inhibitors could be used in the treatment of Parkinson's disease and Alzheimer's disease. Clinically used antidepressants have several limitations and side effects which demand continuous development of novel, efficient and safe drugs for the treatment of depression. ${ }^{5,6}$

In an attempt to synthesize and evaluate new compounds as antidepressant, we report herein, synthesis biological evaluation and docking studies of a number of indole derivatives bearing azetidinone. Indoles are considered as the most promising bicyclic heteroaromatic nucleus in the field of medicinal chemistry. The Indole framework is a medicinally relevant scaffold and has become widely identified as a privileged structure or pharmacophore due to its different pharmacological actions. $7,8,9,10,11$

Literature survey also suggests the potential of the indolyl azetidine-2-one derivatives as anti-inflammatory and antimicrobial agents. ${ }^{12,13}$ Compounds containing the azetidinone nucleus have been reported to possess diverse pharmacological activities. ${ }^{14,15,16,17}$ The indole moiety is present in approved as well as experimental drugs and the azetidinone moiety in experimental drugs. ${ }^{18,19,20}$

Therefore, in the present investigation, it was envisaged that if these two pharmacophores are linked together this would generate new molecular templates Figure 1, which are likely to exhibit antidepressant-like action in animal models. Our research focused on the discovery of new MAO-A inhibitors from the above mentioned

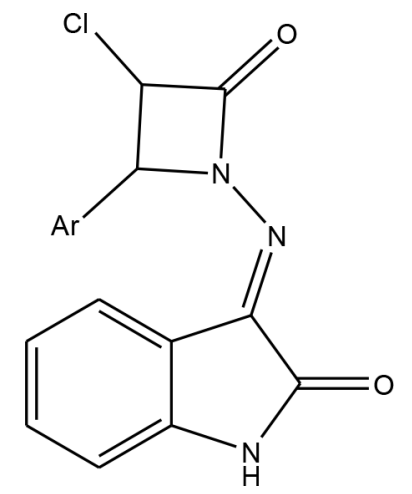

Figure 1: General structure of proposed scaffold as antidepressant agents.

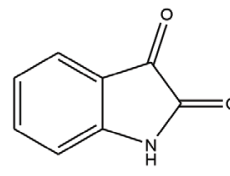

(1)

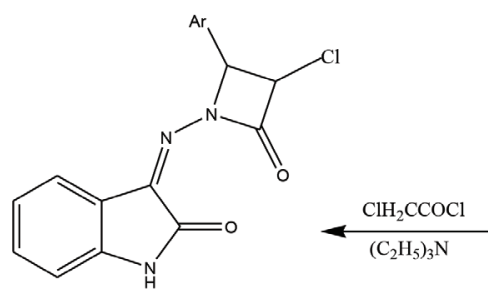

(23-42)

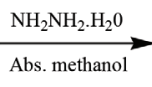

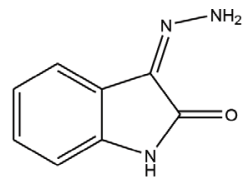

(2)

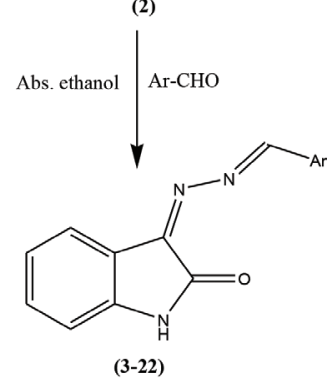

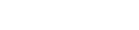

Figure 2: Scheme synthetic route of the titled compounds.

pharmacophores which would be used in the management of depression. A molecular docking study was performed for the prediction of the binding model of the final derivatives in the target of MAO-A enzyme. The synthetic strategy for the final derivatives involved in the reaction between Schiff bases with chloroacetyl chloride in the presence of a catalytic amount of triethylamine. The scheme of the present study is outlined in Figure 2 along with the substitutions shown in Table 1 .

\section{MATERIALS AND METHODS}

\section{Chemistry}

Chemicals were obtained from Sigma-Aldrich (St. Louis, MO, USA), S D Fine-Chem (Mumbai, MH, India) and Merck (Darmstadt, Germany), unless specified. Melting points (m.p.) were detected with open capillaries using ThermoNik precision melting point cum boiling point apparatus (model C-PMB-2, Mumbai, MH, India) and are uncorrected. Infrared (IR) spectra $(\mathrm{KBr})$ were recorded on FTIR-8400s spectrophotometer (Shimadzu, Tokyo, Japan) at the Department of Pharmaceutical Sciences, Rashtrasant Tukadoji Maharai (RTM) Nagpur University. Proton $(1 \mathrm{H})$ and carbon 13 (13C) nuclear magnetic resonance (NMR) were obtained using a Bruker Avance II $400 \mathrm{MHz}$ spectrometer (Billerica, MA, USA), using tetramethylsilane (TMS) as internal standard. All chemical shift values were recorded as $\mathrm{d}$ (ppm), coupling constant value $J$ is measured in hertz, the peaks are presented as s (singlet), $\mathrm{d}$ (doublet), $\mathrm{t}$ (triplet), brs (broad singlet), dd (double doublet), m (multiplet). The purity of compounds was controlled by thin layer chromatography (silica gel HF254e361, type 60, $0.25 \mathrm{~mm}$; Merck, Darmstadt, Germany). Electrospray ionization mass spectrometry (ESI-MS) was recorded at Waters Q-TOF spectrometer (Waters, Milford, MA, USA) at 


\begin{tabular}{|c|c|c|c|c|c|}
\hline \multicolumn{2}{|c|}{ Compd No. } & \multirow{2}{*}{$\begin{array}{c}\mathrm{Ar} \\
\mathrm{C}_{6} \mathrm{H}_{5}-\end{array}$} & \multicolumn{2}{|c|}{ Compd No. } & \multirow{2}{*}{$\frac{\mathrm{Ar}}{4-\mathrm{CH}_{3}-\mathrm{C}_{6} \mathrm{H}_{5}-}$} \\
\hline 3 & 23 & & 13 & 33 & \\
\hline 4 & 24 & $4-\mathrm{Cl}-\mathrm{C}_{6} \mathrm{H}_{5}-$ & 14 & 34 & $2-\mathrm{OCH}_{3}-\mathrm{C}_{6} \mathrm{H}_{5}-$ \\
\hline 5 & 25 & $4-\mathrm{C}_{6} \mathrm{H}_{5}-\mathrm{CH}_{2} \mathrm{O}-\mathrm{C}_{6} \mathrm{H}_{5}-$ & 15 & 35 & $4-\mathrm{OCH}_{3}-\mathrm{C}_{6} \mathrm{H}_{5}-$ \\
\hline 6 & 26 & $2-\mathrm{NO}_{2}-\mathrm{C}_{6} \mathrm{H}_{5}-$ & 16 & 36 & $2-\mathrm{Cl}_{-} \mathrm{C}_{6} \mathrm{H}_{5}-$ \\
\hline 7 & 27 & $4-\mathrm{OH}-\mathrm{C}_{6} \mathrm{H}_{5}-$ & 17 & 37 & $3-\mathrm{Br}-\mathrm{C}_{6} \mathrm{H}_{5}-$ \\
\hline 8 & 28 & $4-\mathrm{OH}, 3-\mathrm{OCH}_{3}-\mathrm{C}_{6} \mathrm{H}_{5}-$ & 18 & 38 & $2,4-\left(\mathrm{OCH}_{3}\right)_{2}-\mathrm{C}_{6} \mathrm{H}_{5}-$ \\
\hline 9 & 29 & $4-\left(\mathrm{CH}_{3}\right)_{2}-\mathrm{N}-\mathrm{C}_{6} \mathrm{H}_{5}-$ & 19 & 39 & $4-\mathrm{NO}_{2}-\mathrm{C}_{6} \mathrm{H}_{5}-$ \\
\hline 10 & 30 & $3-\mathrm{NO}_{2}-\mathrm{C}_{6} \mathrm{H}_{5}-$ & 20 & 40 & $2,3-\left(\mathrm{OCH}_{3}\right)_{2}-\mathrm{C}_{6} \mathrm{H}_{5}-$ \\
\hline 11 & 31 & $3,4,5-\left(\mathrm{OCH}_{3}\right)_{3}-\mathrm{C}_{6} \mathrm{H}_{5}-$ & 21 & 41 & $3-\mathrm{Cl}-\mathrm{C}_{6} \mathrm{H}_{5}-$ \\
\hline 12 & 32 & $2,4-(\mathrm{Cl})_{2}-\mathrm{C}_{6} \mathrm{H}_{5}-$ & 22 & 42 & $\mathrm{C}_{4} \mathrm{H}_{3} \mathrm{O}$ - (2-furyl) \\
\hline
\end{tabular}

the Sophisticated Analytical Instrumentation Facility (SAIF), Punjab University (Chandigarh, PB, India).

\section{Synthesis of 3-hydrazinylidene-1, 3-dihydro-2H- indol-2-one (2)}

A mixture of isatin (1) (1 $\mathrm{mmol})$ and hydrazine hydrate (99\%, $0.055 \mathrm{~g}, 1.1 \mathrm{mmol})$ in absolute methanol $(25 \mathrm{ml})$ was refluxed for $1 \mathrm{~h}$ and then cooled to room temperature. The precipitate of hydrazones was filtered and dried. The crude product was recrystallized from ethanol to give hydrazones (2).

Yield: $70 \%$; mp 248-250 ${ }^{\circ} \mathrm{C}$; $\mathrm{R}_{\mathrm{f}} 0.39$ (Methanol: Toulene 1:4); IR $\left(\mathrm{KBr}, \mathrm{cm}^{-1}\right): 3411-3319,\left(\mathrm{NH}, \mathrm{NH}_{2}\right), 1687$ $(\mathrm{C}=\mathrm{O}), 1609(\mathrm{C}=\mathrm{N}) ;{ }^{1} \mathrm{H}$ NMR (DMSO, ppm): $\delta 6.87-$ 7.37 (m, 4H, Ar-H), 10.57 (s, 2H, NH $)_{2}, 9.39$ (s, 1H, $\mathrm{NH})$; EI-MS: $\mathrm{m} / \mathrm{z}[\mathrm{M}+\mathrm{H}]^{+} 162.24$.

General synthesis of 3-[(substituted benzylidene) hydrazinylidene] 1, 3-dihydro-2H-indol-2-one (3-22)

To a solution of compound $2(0.01 \mathrm{~mol})$ in ethanol $(60 \mathrm{~mL})$, substituted aromatic aldehyde $(0.01 \mathrm{~mol})$ along with few drops of glacial acetic acid were added. Then resulting mixture was refluxed for 7-8 $\mathrm{h}$. The excess of the ethanol was distilled off and the remaining mixture was cooled, poured onto crushed ice and filtered. The crude product was recrystallized from $70 \%$ ethanol.

\section{3-[(Benzylidene) hydrazinylidene] 1, 3-dihydro-} 2H-indol-2-one (3)

Yield: $64 \%$; mp 148-150 ${ }^{\circ} \mathrm{C} ; \mathrm{R}_{\mathrm{f}} 0.37$ (Methanol: Toulene 1:4); IR $\left(\mathrm{KBr}, \mathrm{cm}^{-1}\right): 3295(\mathrm{NH}), 1678(\mathrm{C}=\mathrm{O}), 1598$ $(\mathrm{C}=\mathrm{N}) ;{ }^{1} \mathrm{H}$ NMR (DMSO, ppm): $\delta$ 6.84-7.95 (m, 9H, Ar-H), 10.77 (s, 1H, NH), 8.64 (s, 1H, N=CH); EI-MS: $\mathrm{m} / \mathrm{z}[\mathrm{M}+\mathrm{H}]^{+}$250.26. Anal. Calcd for $\mathrm{C}_{15} \mathrm{H}_{11} \mathrm{~N}_{3} \mathrm{O}$ : C, 72.3; H, 4.41; N, 16.84. Found: C, 71.6; H, 4.12; N, 16.23.
3-[(4-Chlorobenzylidene) hydrazinylidene] 1, 3-dihydro-2H-indol-2-one (4)

Yield: $72 \%$; mp 167-169 ${ }^{\circ} \mathrm{C} ; \mathrm{R}_{\mathrm{f}} 0.35$ (Methanol: Toulene 1:4); IR $\left(\mathrm{KBr}, \mathrm{cm}^{-1}\right): 3332(\mathrm{NH}), 1744(\mathrm{C}=\mathrm{O}), 1535$ $(\mathrm{C}=\mathrm{N}) ;{ }^{1} \mathrm{H}$ NMR (DMSO, ppm): $\delta$ 6.31-8.45 (m, 8H, Ar-H), 10.56 (s, 1H, NH), 9.2 (s, 1H, N=CH); EI-MS: $\mathrm{m} / \mathrm{z}[\mathrm{M}+\mathrm{H}]^{+}$284.76. Anal. Calcd for $\mathrm{C}_{15} \mathrm{H}_{10} \mathrm{ClN}_{3} \mathrm{O}$ : C, 63.6; H, 3.53; N, 14.84. Found: C, 62.6; H, 3.12; N, 13.63 .

\section{3-[(4-Benzoyloxybenzylidene) hydrazinylidene] 1, 3-dihydro-2H-indol-2-one (5)}

Yield: 67\%; mp 143-145 ${ }^{\circ} \mathrm{C} ; \mathrm{R}_{\mathrm{f}} 0.47$ (Methanol: Toulene 1:4); IR (KBr, cm $\left.{ }^{-1}\right): 3327(\mathrm{NH}), 1710(\mathrm{C}=\mathrm{O}), 1534$ $(\mathrm{C}=\mathrm{N}) ;{ }^{1} \mathrm{H}$ NMR (DMSO, ppm): $\delta$ 6.11-7.68 (m, 13H, Ar-H), 8.32 (s, 1H, NH), 8.77 (s, 1H, N=CH); 4.53 (s, $2 \mathrm{H}, \mathrm{CH}_{2}$ ): EI-MS: $\mathrm{m} / \mathrm{z}[\mathrm{M}+\mathrm{H}]^{+}$356.38. Anal. Calcd for $\mathrm{C}_{22} \mathrm{H}_{17} \mathrm{~N}_{3} \mathrm{O}_{2}$ : C, 74.15; H, 4.77; N, 11.78. Found: C, 72.89; H, 4.11; N, 10.35 .

\section{3-[(2-Nitrobenzylidene) hydrazinylidene] 1, 3-dihydro-2H-indol-2-one (6)}

Yield: $64 \%$; mp $210-212{ }^{\circ} \mathrm{C} ; \mathrm{R}_{\mathrm{f}} 0.48$ (Methanol: Toulene 1:4); IR ( $\left.\mathrm{KBr}, \mathrm{cm}^{-1}\right): 3324(\mathrm{NH}), 1724(\mathrm{C}=\mathrm{O}), 1548$ $(\mathrm{C}=\mathrm{N}) ;{ }^{1} \mathrm{H}$ NMR (DMSO, ppm): $\delta$ 6.90-8.28 (m, 8H, Ar-H), 10.89 (s, 1H, NH), 8.82 (s, 1H, N=CH); EI-MS: $\mathrm{m} / \mathrm{z}[\mathrm{M}+\mathrm{H}]^{+}$295.26. Anal. Calcd for $\mathrm{C}_{15} \mathrm{H}_{10} \mathrm{~N}_{4} \mathrm{O}_{3}$ : C, 60.96; H, 3.38; N, 18.96. Found: C, 58.66; H, 3.15; $\mathrm{N}, 17.36$.

\section{3-[(4-Hydroxybenzylidene) hydrazinylidene] 1, 3-dihydro-2H-indol-2-one (7)}

Yield: 58 \%; mp 204-206 ${ }^{\circ} \mathrm{C} ; \mathrm{R}_{\mathrm{f}} 0.43$ (Methanol: Toulene 1:4); IR (KBr, cm $\left.{ }^{-1}\right): 3485(\mathrm{OH}), 3295(\mathrm{NH}), 1716$ $(\mathrm{C}=\mathrm{O}), 1526(\mathrm{C}=\mathrm{N}) ;{ }^{1} \mathrm{H}$ NMR (DMSO, ppm): $\delta 6.87$ 8.10 (m, 8H, Ar-H), 10.78 (s, 1H, NH), 8.53 (s, 1H, 
$\mathrm{N}=\mathrm{CH}) ; 9.44$ (s, 1H, OH); EI-MS: m/z [M+H] 266.26. Anal. Calcd for $\mathrm{C}_{15} \mathrm{H}_{11} \mathrm{~N}_{3} \mathrm{O}$ : C, 67.85; H, 4.14; N, 15.83 . Found: C, 66.61; H, 3.95; N, 15.11

\section{3-[(4-Hydroxy, 3-methoxybenzylidene)} hydrazinylidene] 1, 3-dihydro-2H-indol-2-one (8)

Yield: $64 \%$; mp 184-186 ${ }^{\circ} \mathrm{C}$; $\mathrm{R}_{\mathrm{f}} 0.61$ (Methanol: Toulene 1:4); IR $\left(\mathrm{KBr}, \mathrm{cm}^{-1}\right): 3525(\mathrm{OH}), 3395(\mathrm{NH}), 1693$ $(\mathrm{C}=\mathrm{O}), 1479(\mathrm{C}=\mathrm{N}) ;{ }^{1} \mathrm{H}$ NMR (DMSO, ppm): $\delta$ 6.898.18 (m, 7H, Ar-H), 10.22 (s, 1H, NH), 8.63 (s, 1H, $\mathrm{N}=\mathrm{CH}) ; 3.83$ (s, 3H, $\left.\mathrm{OCH}_{3}\right) ; 9.62$ (s, 1H, OH); EI-MS: $\mathrm{m} / \mathrm{z}[\mathrm{M}+\mathrm{H}]^{+}$266.26. Anal. Calcd for $\mathrm{C}_{15} \mathrm{H}_{11} \mathrm{~N}_{3} \mathrm{O}: \mathrm{C}$, 67.85; H, 4.14; N, 15.83. Found: C, 66.61; H, 3.95; N, 15.11

\section{3-[(4-(Dimethylamino) benzylidene)} hydrazinylidene] 1, 3-dihydro-2H-indol-2-one (9)

Yield: 55\%; mp 164-166 ${ }^{\circ} \mathrm{C} ; \mathrm{R}_{\mathrm{f}} 0.41$ (Methanol: Toulene 1:4); IR ( $\left.\mathrm{KBr}, \mathrm{cm}^{-1}\right): 3345(\mathrm{NH}), 1676(\mathrm{C}=\mathrm{O}), 1523$ $(\mathrm{C}=\mathrm{N}) ;{ }^{1} \mathrm{H}$ NMR (DMSO, ppm): $\delta$ 6.79-8.32 (m, 8H, Ar-H), 10.59 (s, 1H, NH), 8.59 (s, 1H, N=CH); 3.42 (s, $6 \mathrm{H}, \mathrm{CH}_{3}$ ); EI-MS: $\mathrm{m} / \mathrm{z}[\mathrm{M}+\mathrm{H}]^{+}$293.33. Anal. Calcd for $\mathrm{C}_{17} \mathrm{H}_{16} \mathrm{~N}_{4} \mathrm{O}$ : C, 69.86; H, 5.47; N, 19.17. Found: C, 68.12; H, 5.45; N, 18.27.

\section{3-[(3-Nitrobenzylidene) hydrazinylidene] 1,} 3-dihydro-2H-indol-2-one (10)

Yield: 66\%; mp 197-199 ${ }^{\circ} \mathrm{C}$; $\mathrm{R}_{\mathrm{f}} 0.46$ (Methanol: Toulene 1:4); IR (KBr, cm $\left.{ }^{-1}\right): 3293(\mathrm{NH}), 1676(\mathrm{C}=\mathrm{O}), 1534$ $(\mathrm{C}=\mathrm{N}) ;{ }^{1} \mathrm{H}$ NMR (DMSO, ppm): $\delta$ 6.99-8.43 (m, 8H, Ar-H), 10.22 (s, 1H, NH), 8.68 (s, 1H, N=CH); EI-MS: $\mathrm{m} / \mathrm{z}[\mathrm{M}+\mathrm{H}]^{+}$295.26. Anal. Calcd for $\mathrm{C}_{15} \mathrm{H}_{10} \mathrm{~N}_{4} \mathrm{O}_{3}$ : C, 60.96; H, 3.38; N, 18.96. Found: C, 59.66; H, 2.95; N, 17.39.

\section{3-[(3,4,5-Trimethoxybenzylidene)hydrazinylidene]} 1, 3-dihydro-2H-indol-2-one (11)

Yield: 68\%; mp 202-204 ${ }^{\circ} \mathrm{C}$; $\mathrm{R}_{\mathrm{f}} 0.66$ (Methanol: Toulene 1:4); IR (KBr, cm $\left.{ }^{-1}\right): 3265(\mathrm{NH}), 1667$ (C=O), 1567 $(\mathrm{C}=\mathrm{N}) ;{ }^{1} \mathrm{H}$ NMR (DMSO, ppm): $\delta$ 6.88-8.46 (m, 6H, Ar-H), 10.80 (s, 1H, NH), 8.61 (s, 1H, N=CH); 3.81 (s, 9H, $\mathrm{CH}_{3}$ ); EI-MS: $\mathrm{m} / \mathrm{z}[\mathrm{M}+\mathrm{H}]^{+}$340.34. Anal. Calcd for $\mathrm{C}_{18} \mathrm{H}_{17} \mathrm{~N}_{3} \mathrm{O}_{4}: \mathrm{C}, 63.71 ; \mathrm{H}, 5.01 ; \mathrm{N}, 12.38$. Found: C, 62.66; H, 4.95; N, 11.67 .

3-[(2, 4-Dichlorobenzylidene) hydrazinylidene] 1, 3-dihydro-2H-indol-2-one (12)

Yield: 62\%; mp 167-169 ${ }^{\circ} \mathrm{C}$; $\mathrm{R}_{\mathrm{f}} 0.56$ (Methanol: Toulene 1:4); IR ( $\left.\mathrm{KBr}, \mathrm{cm}^{-1}\right): 3353(\mathrm{NH}), 1703$ (C=O), 1610 $(\mathrm{C}=\mathrm{N}) ;{ }^{1} \mathrm{H}$ NMR (DMSO, ppm): $\delta \quad 6.58-8.23$ (m, 7H, Ar-H), 9.45 (s, 1H, NH), 10.86 (s, 1H, N=CH); EI-MS: $\mathrm{m} / \mathrm{z}[\mathrm{M}+\mathrm{H}]^{+}$319.15. Anal. Calcd for $\mathrm{C}_{15} \mathrm{H}_{9} \mathrm{Cl}_{2} \mathrm{~N}_{3} \mathrm{O}: \mathrm{C}$,
56.60; H, 2.83; N, 13.20. Found: C, 55.13; H, 2.31; N, 12.82 .

\section{3-[(4-Methylbenzylidene) hydrazinylidene] 1,} 3-dihydro-2H-indol-2-one (13)

Yield: 71\%; mp 187-189 ${ }^{\circ} \mathrm{C}$; $\mathrm{R}_{\mathrm{f}} 0.36$ (Methanol: Toulene 1:4); IR ( $\left.\mathrm{KBr}, \mathrm{cm}^{-1}\right): 3383(\mathrm{NH}), 1715(\mathrm{C}=\mathrm{O}), 1622$ $(\mathrm{C}=\mathrm{N}) ;{ }^{1} \mathrm{H}$ NMR (DMSO, ppm): $\delta$ 6.90-8.55 (m, 8H, Ar-H), 10.80 (s, $1 \mathrm{H}, \mathrm{NH}), 8.60$ (s, $1 \mathrm{H}, \mathrm{N}=\mathrm{CH}) ; 2.42$ (s, 3H, CH3); EI-MS: m/z [M+H] ${ }^{+}$264.29. Anal. Calcd for $\mathrm{C}_{16} \mathrm{H}_{13} \mathrm{~N}_{3} \mathrm{O}$ : C, 73.02; H, 4.94; N, 15.96. Found: C, 72.13; H, 4.22; N, 14.87.

\section{3-[(2-Methoxybenzylidene) hydrazinylidene] 1, 3-dihydro-2H-indol-2-one (14)}

Yield: 59\%; mp 156-158 ${ }^{\circ} \mathrm{C}$; $\mathrm{R}_{\mathrm{f}} 0.46$ (Methanol: Toulene 1:4); IR $\left(\mathrm{KBr}, \mathrm{cm}^{-1}\right): 3368(\mathrm{NH}), 1740(\mathrm{C}=\mathrm{O}), 1522$ $(\mathrm{C}=\mathrm{N}) ;{ }^{1} \mathrm{H}$ NMR (DMSO, ppm): $\delta$ 6.99-8.21 (m, 8H, Ar-H), 10.75 (s, 1H, NH), 8.95 (s, 1H, N=CH); 3.91 (s, $\left.3 \mathrm{H}, \mathrm{OCH}_{3}\right) ; \mathrm{EI}-\mathrm{MS}: \mathrm{m} / \mathrm{z}[\mathrm{M}+\mathrm{H}]^{+}$280.29. Anal. Calcd for $\mathrm{C}_{16} \mathrm{H}_{13} \mathrm{~N}_{3} \mathrm{O}_{2}$ : C, 68.81; H, 4.65; N, 15.05. Found: C, 67.43; H, 3.28; N, 13.32 .

\section{3-[(4-Methoxybenzylidene) hydrazinylidene] 1, 3-dihydro-2H-indol-2-one (15)}

Yield: $72 \%$; mp 166-168 ${ }^{\circ} \mathrm{C} ; \mathrm{R}_{\mathrm{f}} 0.51$ (Methanol: Toulene 1:4); IR (KBr, $\left.\mathrm{cm}^{-1}\right): 3364(\mathrm{NH}), 1745(\mathrm{C}=\mathrm{O}), 1552$ $(\mathrm{C}=\mathrm{N}) ;{ }^{1} \mathrm{H}$ NMR (DMSO, ppm): $\delta$ 6.23-7.88 (m, 8H, Ar-H), 8.46 (s, 1H, NH), 8.67 (s, 1H, N=CH); 2.50 (s, 3H, OCH3), EI-MS: $\mathrm{m} / \mathrm{z}[\mathrm{M}+\mathrm{H}]^{+}$280.29. Anal. Calcd for $\mathrm{C}_{16} \mathrm{H}_{13} \mathrm{~N}_{3} \mathrm{O}_{2}: \mathrm{C}, 68.81 ; \mathrm{H}, 4.65 ; \mathrm{N}, 15.05$. Found: C, 66.43; H, 4.28; N, 13.72.

\section{3-[(2-Chlorobenzylidene) hydrazinylidene] 1, 3-dihydro-2H-indol-2-one (16)}

Yield: 67\%; mp 189-191 ${ }^{\circ} \mathrm{C}$; $\mathrm{R}_{\mathrm{f}} 0.47$ (Methanol: Toulene 1:4); IR ( $\left.\mathrm{KBr}, \mathrm{cm}^{-1}\right): 3345(\mathrm{NH}), 1678(\mathrm{C}=\mathrm{O}), 1572$ $(\mathrm{C}=\mathrm{N}) ;{ }^{1} \mathrm{H}$ NMR (DMSO, ppm): $\delta$ 6.63-7.81 (m, 8H, Ar-H), 9.34 (s, 1H, NH), 8.55 (s, 1H, N=CH); EI-MS: $\mathrm{m} / \mathrm{z}[\mathrm{M}+\mathrm{H}]^{+}$284.71. Anal. Calcd for $\mathrm{C}_{15} \mathrm{H}_{10} \mathrm{ClN}_{3} \mathrm{O}$ : C, 63.60; H, 3.53; N, 14.84. Found: C, 62.43; H, 3.23; N, 13.68 .

\section{3-[(3-Bromobenzylidene) hydrazinylidene] 1, 3-dihydro-2H-indol-2-one (17)}

Yield: 58\%; mp 176-178 ${ }^{\circ} \mathrm{C} ; \mathrm{R}_{\mathrm{f}} 0.57$ (Methanol: Toulene 1:4); IR $\left(\mathrm{KBr}, \mathrm{cm}^{-1}\right): 3323(\mathrm{NH}), 1658(\mathrm{C}=\mathrm{O}), 1562$ $(\mathrm{C}=\mathrm{N}) ;{ }^{1} \mathrm{H}$ NMR (DMSO, ppm): $\delta$ 6.64-7.71 (m, 8H, Ar-H), 8.33 (s, 1H, NH), 8.89 (s, 1H, N=CH); EI-MS: $\mathrm{m} / \mathrm{z}[\mathrm{M}+\mathrm{H}]^{+}$329.16. Anal. Calcd for $\mathrm{C}_{15} \mathrm{H}_{10} \mathrm{BrN}_{3} \mathrm{O}$ : C, 54.87; H, 3.04; N, 12.80. Found: C, 54.43; H, 2.83; N, 11.68. 
3-[(2, 4-Dimethoxybenzylidene) hydrazinylidene] 1, 3-dihydro-2H-indol-2-one (18)

Yield: 68\%; mp 166-168 ${ }^{\circ} \mathrm{C}$; $\mathrm{R}_{\mathrm{f}} 0.45$ (Methanol: Toulene 1:4); IR ( $\left.\mathrm{KBr}, \mathrm{cm}^{-1}\right): 3298(\mathrm{NH}), 1710(\mathrm{C}=\mathrm{O}), 1555$ $(\mathrm{C}=\mathrm{N}) ;{ }^{1} \mathrm{H}$ NMR (DMSO, ppm): $\delta$ 6.90-8.00 (m, 7H, Ar-H), 10.76 (s, 1H,

$\mathrm{NH}), 8.86$ (s, 1H, N=CH); 3.90 (s, 6H, OCH3); EI-MS: $\mathrm{m} / \mathrm{z}[\mathrm{M}+\mathrm{H}]^{+}$310.31. Anal. Calcd for $\mathrm{C}_{17} \mathrm{H}_{15} \mathrm{~N}_{3} \mathrm{O}_{3}: \mathrm{C}$, $66.01 ; \mathrm{H}, 4.85 ; \mathrm{N}, 13.59$. Found: C, 65.23; H, 4.23; N, 12.45 .

\section{3-[(4-Nitrobenzylidene) hydrazinylidene] 1,} 3-dihydro-2H-indol-2-one (19)

Yield: $57 \%$; mp $165-167^{\circ} \mathrm{C} ; \mathrm{R}_{\mathrm{f}} 0.42$ (Methanol: Toulene 1:4); IR ( $\left.\mathrm{KBr}, \mathrm{cm}^{-1}\right): 3398(\mathrm{NH}), 1704(\mathrm{C}=\mathrm{O}), 1542$ $(\mathrm{C}=\mathrm{N}) ;{ }^{1} \mathrm{H}$ NMR (DMSO, ppm): $\delta$ 6.89-8.39 (m, 8H, Ar-H), 8.63 (s, 1H, NH), 8.63 (s, 1H, N=CH); EI-MS: $\mathrm{m} / \mathrm{z}[\mathrm{M}+\mathrm{H}]^{+}$295.26. Anal. Calcd for $\mathrm{C}_{15} \mathrm{H}_{10} \mathrm{~N}_{4} \mathrm{O}_{3}: \mathrm{C}$, 61.22; H, 3.40; N, 19.04. Found: C, 60.23; H, 2.71; N, 18.35 .

3-[(2, 3-Dimethoxybenzylidene) hydrazinylidene] 1, 3-dihydro-2H-indol-2-one (20)

Yield: 63\%; mp 157-159 ${ }^{\circ} \mathrm{C}$; $\mathrm{R}_{\mathrm{f}} 0.38$ (Methanol: Toulene 1:4); IR (KBr, cm$\left.{ }^{-1}\right)$ : $3354(\mathrm{NH}), 1705(\mathrm{C}=\mathrm{O}), 1532$ $(\mathrm{C}=\mathrm{N}) ;{ }^{1} \mathrm{H}$ NMR (DMSO, ppm): $\delta$ 6.61-8.13 (m, 7H, Ar-H), 10.71 (s, 1H, NH), 8.90 (s, 1H, N=CH); 3.91 (s, 6H, OCH3); EI-MS: $\mathrm{m} / \mathrm{z}[\mathrm{M}+\mathrm{H}]^{+}$310.26. Anal. Calcd for $\mathrm{C}_{15} \mathrm{H}_{10} \mathrm{~N}_{4} \mathrm{O}_{3}: \mathrm{C}, 66.01 ; \mathrm{H}, 4.85 ; \mathrm{N}, 13.59$. Found: $\mathrm{C}$, 65.12; H, 4.43; N, 12.53 .

\section{3-[(3-Chlorobenzylidene) hydrazinylidene] 1, 3-dihydro-2H-indol-2-one (21)}

Yield: 68\%; mp 155-157 ${ }^{\circ} \mathrm{C} ; \mathrm{R}_{\mathrm{f}} 0.41$ (Methanol: Toulene 1:4); IR ( $\left.\mathrm{KBr}, \mathrm{cm}^{-1}\right): 3357(\mathrm{NH}), 1723$ (C=O), 1542 $(\mathrm{C}=\mathrm{N}) ;{ }^{1} \mathrm{H}$ NMR (DMSO, ppm): $\delta$ 6.41-7.41 (m, 8H, Ar-H), 8.41 (s, 1H, NH), 8.93 (s, 1H, N=CH); EI-MS: m/z $[\mathrm{M}+\mathrm{H}]^{+}$284.71. Anal. Calcd for $\mathrm{C}_{15} \mathrm{H}_{10} \mathrm{ClN}_{3} \mathrm{O}$ : C, 63.60; H, 3.53; N, 14.84. Found: C, 62.59; H, 3.12; N, 13.88 .

\section{3-[(Furfuran) hydrazinylidene] 1, 3-dihydro-2H- indol-2-one (22)}

Yield: 64\%; mp 147-149 ${ }^{\circ} \mathrm{C}$; $\mathrm{R}_{\mathrm{f}} 0.37$ (Methanol: Toulene 1:4); IR ( $\left.\mathrm{KBr}, \mathrm{cm}^{-1}\right)$ : $3361(\mathrm{NH}), 1731(\mathrm{C}=\mathrm{O}), 1541$ $(\mathrm{C}=\mathrm{N}) ;{ }^{1} \mathrm{H}$ NMR (DMSO, ppm): $\delta$ 6.11-7.53 (m, 7H, Ar-H), 8.32 (s, 1H, NH), 8.54 (s, 1H, N=CH); EI-MS: m/z $[\mathrm{M}+\mathrm{H}]^{+}$240.26. Anal. Calcd for $\mathrm{C}_{15} \mathrm{H}_{10} \mathrm{~N}_{4} \mathrm{O}_{3}$ : C, 75.12; H, 4.16; N, 23.33. Found: C, 74.65; H, 3.78; N, 22.78.

General synthesis of $3\{[3-c h l o r o-2-s u b s t i t u t e d-4-$ oxoazetidin-1-yl] imino\}-1, 3-dihydro-2H-indol-2one (23-42)

A mixture of Schiff base (3-22) $(0.01 \mathrm{~mol})$ and triethyl amine (0.02 mol) was dissolved in 1, 4-Dioxane (15 m1).
To this, a solution of chloroacetyl chloride $(0.02 \mathrm{~mol})$ was added in portion wise with vigorous shaking at room temperature for $20 \mathrm{~min}$. The reaction mixture was heated under reflux for $3 \mathrm{~h}$ and the content was kept at room temperature for $48 \mathrm{~h}$ and poured into ice-cold water. The resulting solid was filtered, washed several times with water and then recrystalised from $70 \%$ ethanol.

3\{[3-Chloro-4-oxo-2-phenylazetidin-1-yl] imino\}-1, 3-dihydro-2H-indol-2-one (23)

Yield: 63\%; mp 172-174 ${ }^{\circ} \mathrm{C}$; $\mathrm{R}_{f} 0.53$ (Methanol: Toulene 1:4); IR ( $\left.\mathrm{KBr}, \mathrm{cm}^{-1}\right)$ : $3367(\mathrm{NH}), 1704(\mathrm{C}=\mathrm{O}), 1542$ $(\mathrm{C}=\mathrm{N}) ;{ }^{1} \mathrm{H}$ NMR (DMSO, ppm): $\delta$ 7.03-8.37 (m, 9H, Ar-H), 8.66 (s, 1H, NH), 6.88 (d, 1H, CH-Ar); 5.48 (d, 1H, CH-Cl); EI-MS: $\mathrm{m} / \mathrm{z}[\mathrm{M}+\mathrm{H}]^{+}$326.74. Anal. Calcd for $\mathrm{C}_{17} \mathrm{H}_{12} \mathrm{ClN}_{3} \mathrm{O}_{2}: \mathrm{C}, 62.76 ; \mathrm{H}, 3.69 ; \mathrm{N}, 12.92$. Found: $\mathrm{C}$, 61.45; H, 2.79; N, 11.77.

\section{3\{[3-Chloro-2-(4-chlorophenyl) -4-oxoazetidin-1-} yl] imino\}-1, 3-dihydro-2H-indol-2-one (24)

Yield: 71\%; mp 191-193 ${ }^{\circ} \mathrm{C} ; \mathrm{R}_{\mathrm{f}} 0.61$ (Methanol: Toulene 1:4); IR (KBr, cm$\left.{ }^{-1}\right): 3369$ (NH), 1699 (C=O), 1521 $(\mathrm{C}=\mathrm{N}) ;{ }^{1} \mathrm{H}$ NMR (DMSO, ppm): $\delta$ 7.10-7.95 (m, 8H, Ar-H), 8.65 (s, 1H, NH), 5.88 (d, 1H, CH-Ar); 5.04 (d, 1H, CH-Cl); EI-MS: $\mathrm{m} / \mathrm{z}[\mathrm{M}+\mathrm{H}]^{+}$361.94. Anal. Calcd for $\mathrm{C}_{17} \mathrm{H}_{11} \mathrm{Cl}_{2} \mathrm{~N}_{3} \mathrm{O}_{2}$ : C, 56.66; $\mathrm{H}, 3.05 ; \mathrm{N}, 11.66$. Found: C, 55.21; H, 2.79; N, 10.75.

$3\{$ [ 3 - C h l or o- 2 - ( 4 - be nzo y l ox y phen y I) -4-oxoazetidin-1-yl] imino\}-1, 3-dihydro-2H-indol2-one (25)

Yield: $61 \%$; mp 135-137 ${ }^{\circ} \mathrm{C}$; $\mathrm{R}_{\mathrm{f}} 0.55$ (Methanol: Toulene 1:4); IR ( $\left.\mathrm{KBr}, \mathrm{cm}^{-1}\right)$ : $3267(\mathrm{NH}), 1698(\mathrm{C}=\mathrm{O}), 1545$ $(\mathrm{C}=\mathrm{N}) ;{ }^{1} \mathrm{H}$ NMR (DMSO, ppm): $\delta$ 7.05-8.89 (m, $13 \mathrm{H}$, Ar-H), 9.56 (s, 1H, NH), 4.78 (d, 1H, CH-Ar); 6.12 (d, 1H, CH-Cl); 5.20 (d, 2H, $\mathrm{CH}_{2}$ ); EI-MS: $\mathrm{m} / \mathrm{z}[\mathrm{M}+\mathrm{H}]^{+}$ 432.87. Anal. Calcd for $\mathrm{C}_{24} \mathrm{H}_{18} \mathrm{ClN}_{3} \mathrm{O}_{3}: \mathrm{C}, 66.70 ; \mathrm{H}, 4.16$; N, 9.72. Found: C, 65.61; H, 3.89; N, 9.15.

3\{[3-Chloro-2-(2-nitrophenyl)-4-oxoazetidin-1-yl] imino\}-1, 3-dihydro-2H-indol-2-one (26)

Yield: $63 \%$; mp $168-170^{\circ} \mathrm{C} ; \mathrm{R}_{\mathrm{f}} 0.42$ (Methanol: Toulene 1:4); IR ( $\left.\mathrm{KBr}, \mathrm{cm}^{-1}\right): 3395$ (NH), 1675 (C=O), 1586 $(\mathrm{C}=\mathrm{N}) ;{ }^{1} \mathrm{H}$ NMR (DMSO, ppm): $\delta$ 6.84-8.26 (m, 8H, Ar-H), 8.97 (s, 1H, NH), 5.07 (d, 1H, CH-Ar); 5.49 (d, 1H, CH-Cl); EI-MS: m/z $[\mathrm{M}+\mathrm{H}]^{+}$371.74. Anal. Calcd for $\mathrm{C}_{17} \mathrm{H}_{11} \mathrm{ClN}_{4} \mathrm{O}_{4}$ : C, 55.13; H, 2.97; N, 15.13 . Found: C, 54.33; H, 1.97; N, 14.94 .

$3\{[3-C h l o r o-2-(4-h y d r o x y p h e n y l)$-4-oxoazetidin-1yl] imino\}-1, 3-dihydro-2H-indol-2-one (27)

Yield: $72 \%$; mp $211-213^{\circ} \mathrm{C}$; $\mathrm{R}_{\mathrm{f}} 0.58$ (Methanol: Toulene 1:4); IR ( $\left.\mathrm{KBr}, \mathrm{cm}^{-1}\right): 3324(\mathrm{NH}), 1723(\mathrm{C}=\mathrm{O}), 1576$ $(\mathrm{C}=\mathrm{N}) ;{ }^{1} \mathrm{H}$ NMR (DMSO, ppm): $\delta$ 7.66-7.92 (m, 8H, 
Ar-H), 8.45 (s, 1H, NH), 5.66 (d, 1H, CH-Ar); 6.71 (d, 1H, CH-Cl); 9.56 (s, 1H, OH); EI-MS: m/z [M+H] 342.74. Anal. Calcd for $\mathrm{C}_{17} \mathrm{H}_{12} \mathrm{ClN}_{3} \mathrm{O}_{3}$ : C, 59.82; $\mathrm{H}, 3.51$; N, 12.31. Found: C, 58.51; H, 2.98; N, 11.87.

\section{3\{[3-Chloro-2-(4-hydroxy, 3-methoxy phenyl) -4-oxoazetidin-1-yl]imino\}-1,3-dihydro-2H-indol-2- one (28)}

Yield: $67 \%$; mp 178- $180^{\circ} \mathrm{C} ; \mathrm{R}_{\mathrm{f}} 0.44$ (Methanol: Toulene 1:4); IR $\left(\mathrm{KBr}, \mathrm{cm}^{-1}\right): 3289(\mathrm{NH}), 1689(\mathrm{C}=\mathrm{O}), 1557(\mathrm{C}=\mathrm{N})$; ${ }^{1} \mathrm{H}$ NMR (DMSO, ppm): $\delta$ 7.72-8.02 (m, 7H, Ar-H), 8.75 (s, 1H, NH), 5.21 (d, 1H, CH-Ar); 6.55 (d, 1H, $\mathrm{CH}-\mathrm{Cl}) ; 2.76$ (s, 3H, $\left.\mathrm{OCH}_{3}\right) ; 9.87$ (s, 1H, OH); EI-MS: $\mathrm{m} / \mathrm{z}[\mathrm{M}+\mathrm{H}]^{+}$372.77. Anal. Calcd for $\mathrm{C}_{18} \mathrm{H}_{14} \mathrm{ClN}_{3} \mathrm{O}_{4}$ : C, 58.22; H, 3.77; N, 11.32. Found: C, 57.45; H, 2.94; N, 10.92 .

\section{3\{[3-Chloro-2-(4-(Dimethylamino) phenyl)} -4-oxoazetidin-1-yl] imino\}-1, 3-dihydro-2H-indol2-one (29)

Yield: $76 \%$; mp 182-184 ${ }^{\circ} \mathrm{C} ; \mathrm{R}_{\mathrm{f}} 0.61$ (Methanol: Toulene 1:4); IR ( $\left.\mathrm{KBr}, \mathrm{cm}^{-1}\right)$ : 3295 (NH), 1745 (C=O), 1577 $(\mathrm{C}=\mathrm{N}) ;{ }^{1} \mathrm{H}$ NMR (DMSO, ppm): $\delta$ 6.76-8.70 (m, 8H, Ar-H), 10.72 (s, 1H, NH), 5.05 (d, 1H, CH-Ar); 5.45 (d, $1 \mathrm{H}, \mathrm{CH}-\mathrm{Cl}$ ); 3.57 (s, 6H, $\mathrm{CH}_{3}$ ); EI-MS: m/z [M+H] 369.81. Anal. Calcd for $\mathrm{C}_{19} \mathrm{H}_{17} \mathrm{ClN}_{4} \mathrm{O}_{2}: \mathrm{C}, 61.95 ; \mathrm{H}, 4.61$; N, 15.21. Found: C, 60.12; H, 4.12; N, 14.63.

3\{[3-Chloro-2-(3-nitrophenyl) -4-oxoazetidin-1-yl] imino\}-1, 3-dihydro-2H-indol-2-one (30)

Yield: 58\%; mp 204-206 ${ }^{\circ} \mathrm{C}$; $\mathrm{R}_{\mathrm{f}} 0.43$ (Methanol: Toulene 1:4); IR (KBr, cm$\left.{ }^{-1}\right): 3292(\mathrm{NH}), 1672$ (C=O), 1543 $(\mathrm{C}=\mathrm{N}) ;{ }^{1} \mathrm{H}$ NMR (DMSO, ppm): $\delta$ 7.78-8.09 (m, 8H, Ar-H), 9.22 (s, 1H, NH), 5.23 (d, 1H, CH-Ar); 5.98 (d, 1H, CH-Cl); EI-MS: m/z $[\mathrm{M}+\mathrm{H}]^{+}$371.74. Anal. Calcd for $\mathrm{C}_{17} \mathrm{H}_{11} \mathrm{ClN}_{4} \mathrm{O}_{4}$ : C, 55.13; H, 2.97; N, 15.13 . Found: C, 54.37; H, 2.56; N, 14.61.

3\{[3-Chloro-2-(3, 4, 5-trimethoxyphenyl)-4oxoazetidin-1-yl] imino\}-1, 3-dihydro-2H-indol-2one (31)

Yield: $61 \%$; mp 221-223 ${ }^{\circ} \mathrm{C}$; $\mathrm{R}_{\mathrm{f}} 0.52$ (Methanol: Toulene 1:4); IR (KBr, cm$\left.{ }^{-1}\right): 3342(\mathrm{NH}), 1626(\mathrm{C}=\mathrm{O}), 1537$ $(\mathrm{C}=\mathrm{N}) ;{ }^{1} \mathrm{H}$ NMR (DMSO, ppm): $\delta$ 7.66-8.16 (m, 6H, Ar-H), 8.78 (s, 1H, NH), 5.43 (d, 1H, CH-Ar); 5.51 (d, 1H, CH-Cl); 3.93 (s, 9H, $\mathrm{CH}_{3}$ ); EI-MS: m/z [M+H] 416.82. Anal. Calcd for $\mathrm{C}_{20} \mathrm{H}_{18} \mathrm{ClN}_{3} \mathrm{O}_{5}: \mathrm{C}, 57.83 ; \mathrm{H}, 4.33$; N, 10.12. Found: C, 56.37; H, 4.12; N, 9.82.

3\{[3-Chloro-2-(2, 4-dichlorophenyl)-4-oxoazetidin1-yl] imino\}-1, 3-dihydro-2H-indol-2-one (32)

Yield: 64\%; mp 224-226 ${ }^{\circ} \mathrm{C}$; $\mathrm{R}_{\mathrm{f}} 0.62$ (Methanol: Toulene 1:4); IR ( $\left.\mathrm{KBr}, \mathrm{cm}^{-1}\right): 3286(\mathrm{NH}), 1723(\mathrm{C}=\mathrm{O}), 1529$ $(\mathrm{C}=\mathrm{N}) ;{ }^{1} \mathrm{H}$ NMR (DMSO, ppm): $\delta$ 7.23-8.15 (m, 7H,
Ar-H), 9.45 (s, 1H, NH), 5.23 (d, 1H, CH-Ar); 5.03 (d, 1H, CH-Cl); EI-MS: $\mathrm{m} / \mathrm{z}[\mathrm{M}+\mathrm{H}]^{+}$395.63. Anal. Calcd for $\mathrm{C}_{17} \mathrm{H}_{10} \mathrm{Cl}_{3} \mathrm{~N}_{3} \mathrm{O}_{2}: \mathrm{C}, 51.77 ; \mathrm{H}, 2.53 ; \mathrm{N}, 10.65$. Found: C, 50.45; H, 1.98; N, 9.72 .

3\{[3-Chloro-2-(4-Methylphenyl) -4-oxoazetidin-1yl] imino\}-1, 3-dihydro-2H-indol-2-one (33)

Yield: 61\%; mp 173-175 ${ }^{\circ} \mathrm{C}$; $\mathrm{R}_{\mathrm{f}} 0.57$ (Methanol: Toulene 1:4); IR ( $\left.\mathrm{KBr}, \mathrm{cm}^{-1}\right)$ : $3294(\mathrm{NH}), 1741$ (C=O), 1521 $(\mathrm{C}=\mathrm{N}) ;{ }^{1} \mathrm{H}$ NMR (DMSO, ppm): $\delta 7.34-8.51$ (m, 8H, Ar-H), 9.23 (s, 1H, NH), 5.13 (d, 1H, CH-Ar); 5.43 (d, $1 \mathrm{H}, \mathrm{CH}-\mathrm{Cl}$ ); 2.42 (s, 3H, $\mathrm{CH}_{3}$ ); EI-MS: $\mathrm{m} / \mathrm{z}[\mathrm{M}+\mathrm{H}]^{+}$ 340.77. Anal. Calcd for $\mathrm{C}_{18} \mathrm{H}_{14} \mathrm{ClN}_{3} \mathrm{O}_{2}$ : C, 63.71; H, 4.12; N, 12.38. Found: C, 63.53; H, 3.23; N, 11.43.

3\{[3-Chloro-2-(2-Methoxyphenyl) -4-oxoazetidin1-yl] imino\}-1, 3-dihydro-2H-indol-2-one (34)

Yield: 69\%; mp 166-168 ${ }^{\circ} \mathrm{C} ; \mathrm{R}_{\mathrm{f}} 0.41$ (Methanol: Toulene 1:4); IR (KBr, cm $\left.{ }^{-1}\right)$ : $3334(\mathrm{NH}), 1698$ (C=O), 1543 $(\mathrm{C}=\mathrm{N}) ;{ }^{1} \mathrm{H}$ NMR (DMSO, ppm): $\delta$ 6.89-7.81 (m, 8H, Ar-H), 8.99 (s, 1H, NH), 5.07 (d, 1H, CH-Ar); 5.65 (d, 1H, CH-Cl); 2.31 (s, 3H, OCH ${ }_{3}$; EI-MS: m/z [M+H] ${ }^{+}$ 356.77. Anal. Calcd for $\mathrm{C}_{18} \mathrm{H}_{14} \mathrm{ClN}_{3} \mathrm{O}_{3}$ : C, 60.84; H, 3.94; N, 11.83. Found: C, 59.57; H, 2.53; N, 10.43.

$3\{[3-C h l o r o-2-(4-M e t h o x y p h e n y l)$-4-oxoazetidin1-yl] imino\}-1, 3-dihydro-2H-indol-2-one (35)

Yield: 61\%; mp 172-174 ${ }^{\circ} \mathrm{C} ; \mathrm{R}_{\mathrm{f}} 0.54$ (Methanol: Toulene 1:4); IR $\left(\mathrm{KBr}, \mathrm{cm}^{-1}\right): 3296(\mathrm{NH}), 1679(\mathrm{C}=\mathrm{O}), 1521$ $(\mathrm{C}=\mathrm{N}) ;{ }^{1} \mathrm{H}$ NMR (DMSO, ppm): $\delta$ 6.89-8.61 (m, 8H, Ar-H), 10.88 (s, 1H, NH), 5.03 (d, 1H, CH-Ar); 5.34 (d, $1 \mathrm{H}, \mathrm{CH}-\mathrm{Cl}) ; 3.61$ (s, $\left.3 \mathrm{H}, \mathrm{OCH}_{3}\right) ; \mathrm{EI}-\mathrm{MS}: \mathrm{m} / \mathrm{z}[\mathrm{M}+\mathrm{H}]^{+}$ 356.77. Anal. Calcd for $\mathrm{C}_{18} \mathrm{H}_{14} \mathrm{ClN}_{3} \mathrm{O}_{3}$ : C, 60.84; H, 3.94; N, 11.83. Found: C, 59.77; H, 2.61; N, 10.52.

3\{[3-Chloro-2-(2-chlorophenyl) -4-oxoazetidin-1yl] imino\}-1, 3-dihydro-2H-indol-2-one (36)

Yield: $81 \%$; mp 158-160 ${ }^{\circ} \mathrm{C} ; \mathrm{R}_{\mathrm{f}} 0.61$ (Methanol: Toulene 1:4); IR (KBr, cm $\left.{ }^{-1}\right): 3345(\mathrm{NH}), 1709$ (C=O), 1582 $(\mathrm{C}=\mathrm{N}) ;{ }^{1} \mathrm{H}$ NMR (DMSO, ppm): $\delta$ 6.90-8.98 (m, 8H, Ar-H), 10.97 (s, 1H, NH), 5.48 (d, 1H, CH-Ar); 6.91 (d, 1H, CH-Cl); EI-MS: $\mathrm{m} / \mathrm{z}[\mathrm{M}+\mathrm{H}]^{+}$361.19. Anal. Calcd for $\mathrm{C}_{17} \mathrm{H}_{11} \mathrm{Cl}_{2} \mathrm{~N}_{3} \mathrm{O}_{2}$ : C, 56.66; H, 3.05; N, 11.66. Found: C, 55.51; H, 2.61; N, 10.72.

3\{[3-Chloro-2-(3-bromophenyl) -4-oxoazetidin-1yl] imino\}-1, 3-dihydro-2H-indol-2-one (37)

Yield: 67\%; mp 182-184 ${ }^{\circ} \mathrm{C} ; \mathrm{R}_{\mathrm{f}} 0.63$ (Methanol: Toulene 1:4); IR (KBr, cm $\left.{ }^{-1}\right): 3297$ (NH), 1739 (C=O), 1567 $(\mathrm{C}=\mathrm{N}) ;{ }^{1} \mathrm{H}$ NMR (DMSO, ppm): $\delta$ 7.15-8.65 (m, 8H, Ar-H), 9.77 (s, 1H, NH), 5.16 (d, 1H, CH-Ar); 6.32 (d, 1H, CH-Cl); EI-MS: m/z $[\mathrm{M}+\mathrm{H}]^{+}$405.64. Anal. 
Calcd for $\mathrm{C}_{17} \mathrm{H}_{11} \mathrm{BrClN}_{3} \mathrm{O}_{2}$ : C, 50.49; H, 2.72; N, 10.39 . Found: C, 49.32; H, 1.75; N, 9.84 .

$3\{$ [ 3 - Ch l oro-2 - (2 . 4-dimethoxyphenyl) -4-oxoazetidin-1-yl] imino\}-1, 3-dihydro-2H-indol2-one (38)

Yield: $73 \%$; mp 156-158 ${ }^{\circ} \mathrm{C}$; $\mathrm{R}_{\mathrm{f}} 0.43$ (Methanol: Toulene 1:4); IR ( $\left.\mathrm{KBr}, \mathrm{cm}^{-1}\right): 3345(\mathrm{NH}), 1716$ (C=O), 1513 $(\mathrm{C}=\mathrm{N}) ;{ }^{1} \mathrm{H}$ NMR (DMSO, ppm): $\delta$ 6.48-8.92 (m, 7H, Ar-H), 10.42 (s, 1H, NH), 6.13 (d, 1H, CH-Ar); 5.89 (d, 1H, CH-Cl); 3.87 (s, 6H, OCH $)$; EI-MS: m/z [M+H] 386.80. Anal. Calcd for $\mathrm{C}_{19} \mathrm{H}_{16} \mathrm{ClN}_{3} \mathrm{O}_{4}: \mathrm{C}, 59.22 ; \mathrm{H}, 4.15$; N, 10.90. Found: C, 58.59; H, 3.98; N, 9.55.

\section{$3\{[3-C h l o r o-2-(4-n i t r o p h e n y l)$-4-oxoazetidin-1-yl] imino\}-1, 3-dihydro-2H-indol-2-one (39)}

Yield: 59\%; mp 165-167 ${ }^{\circ} \mathrm{C}$; $\mathrm{R}_{\mathrm{f}} 0.65$ (Methanol: Toulene 1:4); IR ( $\left.\mathrm{KBr}, \mathrm{cm}^{-1}\right): 3298(\mathrm{NH}), 1675$ (C=O), 1572 $(\mathrm{C}=\mathrm{N}) ;{ }^{1} \mathrm{H}$ NMR (DMSO, ppm): $\delta$ 7.07-8.87 (m, 8H, Ar-H), 9.33 (s, 1H, NH), 5.31 (d, 1H, CH-Ar); 6.04 (d, 1H, CH-Cl); EI-MS: $\mathrm{m} / \mathrm{z}[\mathrm{M}+\mathrm{H}]^{+}$371.74. Anal. Calcd for $\mathrm{C}_{17} \mathrm{H}_{11} \mathrm{ClN}_{4} \mathrm{O}_{4}$ : C, 55.13; H, 2.97; N, 15.13 . Found: C, 54.65; H, 1.87; N, 14.75.

\section{3\{[3-Chloro-2-(2, 3-dimethoxyphenyl) -4-oxoazetidin-1-yl] imino\}-1, 3-dihydro-2H-indol- 2-one (40)}

Yield: 59\%; mp 165-167 ${ }^{\circ} \mathrm{C} ; \mathrm{R}_{\mathrm{f}} 0.65$ (Methanol: Toulene 1:4); IR ( $\left.\mathrm{KBr}, \mathrm{cm}^{-1}\right): 3298$ (NH), 1675 (C=O), 1572 $(\mathrm{C}=\mathrm{N}) ;{ }^{1} \mathrm{H}$ NMR (DMSO, ppm): $\delta$ 7.12-8.91 (m, 7H, Ar-H), 9.51 (s, 1H, NH), 4.98 (d, 1H, CH-Ar); 6.13 (d, $1 \mathrm{H}, \mathrm{CH}-\mathrm{Cl}) ; 2.41$ (s, $\left.6 \mathrm{H}, \mathrm{OCH}_{3}\right)$; EI-MS: m/z [M+H] 386.80. Anal. Calcd for $\mathrm{C}_{19} \mathrm{H}_{16} \mathrm{ClN}_{3} \mathrm{O}_{4}: \mathrm{C}, 59.09 ; \mathrm{H}, 4.14$; N, 10.88. Found: C, 58.68; H, 3.87; N, 10.11.

\section{3\{[3-Chloro-2-(3-chlorophenyl) -4-oxoazetidin-1- yl] imino\}-1, 3-dihydro-2H-indol-2-one (41)}

Yield: $71 \%$; mp 172-174 ${ }^{\circ} \mathrm{C} ; \mathrm{R}_{\mathrm{f}} 0.43$ (Methanol: Toulene 1:4); IR (KBr, cm$\left.{ }^{-1}\right): 3223(\mathrm{NH}), 1702$ (C=O), 1573 $(\mathrm{C}=\mathrm{N}) ;{ }^{1} \mathrm{H}$ NMR (DMSO, ppm): $\delta$ 7.09-8.78 (m, 8H, Ar-H), 9.23 (s, 1H, NH), 5.34 (d, 1H, CH-Ar); 6.13 (d, 1H, CH-Cl); EI-MS: $\mathrm{m} / \mathrm{z}[\mathrm{M}+\mathrm{H}]^{+}$361.19. Anal. Calcd for $\mathrm{C}_{17} \mathrm{H}_{11} \mathrm{Cl}_{2} \mathrm{~N}_{3} \mathrm{O}_{2}$ : C, 59.09; H, 3.09; N, 11.66. Found: C, 58.65; H, 2.87; N, 11.02 .

\section{$3\{[3-C h l o r o-2-(f u r f u r a n)$-4-oxoazetidin-1-yl] imino\}-1, 3-dihydro-2H-indol-2-one (42)}

Yield: 59\%; mp 165-167 ${ }^{\circ} \mathrm{C}$; $\mathrm{R}_{\mathrm{f}} 0.65$ (Methanol: Toulene 1:4); IR ( $\left.\mathrm{KBr}, \mathrm{cm}^{-1}\right): 3298(\mathrm{NH}), 1675$ (C=O), 1572 $(\mathrm{C}=\mathrm{N}) ;{ }^{1} \mathrm{H}$ NMR (DMSO, ppm): $\delta$ 7.12-8.82 (m, 7H, Ar-H), 9.13 (s, 1H, NH), 5.21 (d, 1H, CH-Ar); 5.88 (d, 1H, CH-Cl); EI-MS: m/z $[\mathrm{M}+\mathrm{H}]^{+}$316.71. Anal. Calcd for $\mathrm{C}_{15} \mathrm{H}_{10} \mathrm{ClN}_{3} \mathrm{O}_{3}: \mathrm{C}, 57.09 ; \mathrm{H}, 3.16 ; \mathrm{N}, 13.30$. Found: C, 56.87; H, 2.87; N, 12.85.

\section{Pharmacology}

The experimental protocols for the pharmacological screening on mice were done with Institutional Animal Ethics Committee, Department of Pharmaceutical Sciences, Rashtrasant Tukadoji Maharaj (RTM) Nagpur University,Nagpur,India(Reg.no.:IAEC/UDPS/2016/21).

\section{Antidepressant activity}

The final synthesized derivatives (23-42) were evaluated for their antidepressant activity by using forced swimming test (behavioural despair test). ${ }^{21}$ in mice at dose of $100 \mathrm{mg} / \mathrm{kg}$ and compared with standard drug fluoxetine $(20 \mathrm{mg} / \mathrm{kg})$. Adult male $(20 \pm 4 \mathrm{~g})$ mice were used with free access to food and water. They were housed in a group of six. Acute oral toxicity of synthesized compounds was determined by OECD guideline no. 425 (up and down procedure). LD50 was calculated as per OECD guidelines 425. ${ }^{22}$ The synthesized compounds $(100 \mathrm{mg} / \mathrm{kg}$ ) and fluoxetine $(20 \mathrm{mg} / \mathrm{kg})$ suspended in aqueous tween $80(0.5 \%)$, were injected as intraperitonealy (i.p.) $(n=6)$. After $1 / 2 \mathrm{hr}$, the mouse was dropped into the glass cylindrical container (diameter $10 \mathrm{~cm}$, height $25 \mathrm{~cm}$ ), containing approximately $20 \mathrm{~cm}$ of water at $25 \pm 1 \circ \mathrm{C}$ temperature. Water was replaced between every trial. Each mouse left for $6 \mathrm{~min}$ at the end of the first $2 \mathrm{~min}$; the animals showing initial vigorous struggling were immobile. The immobility times of each mouse were measured over the period of $4 \mathrm{~min}$. Each mouse was judged immobile when it ceased struggling and remained floating motionless in the water, making only those movements necessary to keep its head above water. Conventional antidepressants decreased the immobility time in this test. Statistical analysis was performed by one-way ANOVA followed by Dunnett's test to evaluate the results. Percentage decrease in immobility duration (\%DID) for test and standard drugs was calculated using following formula:

$$
\% \mathrm{DID}=[\mathrm{A}-\mathrm{B} / \mathrm{B}]^{* 100}
$$

Where $\mathrm{A}$ is the duration of immobility (s) in control group and B is the duration of immobility (s) in test group. All the results of the antidepressant activity are given in Table 2.

\section{Molecular docking study}

For docking purpose, the three-dimensional structure of MAO-A (protein data bank, PDB code 2BXS) was obtained from RCSB PDB. The receptor molecule was refined and validated using Biopredicta $(\mathrm{C}$ module on the Vlife MDS Molecular Modelling software, version 4.3.1. The Vlife MDS suit uses k-nearest neighbour genetic algorithm (KNN-GA) method for molecular 


\begin{tabular}{|c|c|c|c|}
\hline Sr. no. & Compounds & $\begin{array}{c}\text { Duration of immobility } \\
\text { (sec.) } \\
\text { (mean } \pm \text { SEM) }\end{array}$ & $\begin{array}{c}\text { Percentage decrease in } \\
\text { immobility duration } \\
\text { (\% DID) }\end{array}$ \\
\hline 1. & 23 & $54.75 \pm 3.1$ & 46.97 \\
\hline 2. & 24 & $41.75 \pm 2.8$ & 59.56 \\
\hline 3. & 25 & $72.25 \pm 5.5$ & 30.02 \\
\hline 4. & 26 & $34.25 \pm 5.4$ & 66.82 \\
\hline 5. & 27 & $72.75 \pm 6.4$ & 29.53 \\
\hline 6. & 28 & $52.00 \pm 4.7$ & 49.63 \\
\hline 7. & 29 & $55.50 \pm 4.2$ & 46.24 \\
\hline 8. & 30 & $55.75 \pm 3.4$ & 46.00 \\
\hline 9. & 31 & $74.00 \pm 3.0$ & 28.32 \\
\hline 10. & 32 & $63.00 \pm 2.8$ & 38.98 \\
\hline 11. & 33 & $52.50 \pm 2.1$ & 49.15 \\
\hline 12. & 34 & $74.00 \pm 4.5$ & 28.32 \\
\hline 13. & 35 & $79.75 \pm 4.3$ & 22.7 \\
\hline 14. & 36 & $35.50 \pm 3.9$ & 65.61 \\
\hline 15. & 37 & $38.75 \pm 6.2$ & 62.46 \\
\hline 16. & 38 & $57.75 \pm 4.7$ & 44.06 \\
\hline 17. & 39 & $43.25 \pm 1.9$ & 58.11 \\
\hline 18. & 40 & $62.00 \pm 7.2$ & 39.95 \\
\hline 19. & 41 & $42.50 \pm 2.1$ & 58.83 \\
\hline 20. & 42 & $67.75 \pm 4.1$ & 34.38 \\
\hline 21. & Vehicle/control & $103.25 \pm 3.7$ & 0.0 \\
\hline 22. & Fluoxetine $(20 \mathrm{mg} / \mathrm{kg})$ & $30 \pm 1.7$ & 70.94 \\
\hline
\end{tabular}

aData analyzed by one-way ANOVA followed by Dunnett's test. $n=6$; dose $=100 \mathrm{mg} / \mathrm{kg}$. Values are represented as mean \pm S.E.M. Values are significant at $* * * P<$ 0.001 , compared with control group.

docking. The scoring function is based on the basis of best suited moiety for the target with respect to energy and inter molecular interactions. The ligands that were already present within the receptor in bound form were removed to allow for docking protocol. All the ligands were prepared and docked for this study in flexible docking mode and atoms located within a range of $5.0 \AA$ from the amino acid residues were selected in the active site. Standard drug fluoxetine was also included into the series of compounds under study to make comparison with respect to in silico analysis. The docking calculations and energy minimization were set in the Biopredicta $(\mathrm{C}$ module, most of the parameters were set default with 10000 cycles per molecule for the active site cavity No. 1 . The active sites of the MAO-A (PDB code 2BXS) were identified which was comprised ALA44, ALA68, ARG45, ARG51, GLU43, GLY20, GLY22, GLY50, GLY66, HIS242, ILE19, ILE2, ILE273, LEU42, PRO243, SER24, TRP397, TYR69 and TYR402 that were the interacting residues.

\section{RESULTS AND DISCUSSION}

\section{Chemistry}

The reported investigation deals with synthesis and characterization of several indole bearing azetidinone nucleuses to form final twenty derivatives. To achieve these, three different steps were carried out. In the first step, isatin ( $1 H$-indole-2,3-dione) 1 was reacted with hydrazine hydrate in presence of methanol under conditions of reflux to yield the 3-hydrazinylidene1,3-dihydro- $2 H$-indol-2-one 2. NMR spectra of this compound exhibited prominent signals at $\delta 9.39 \mathrm{ppm}$ and $10.57 \mathrm{ppm}$ corresponding to the secondary amide proton and primary amine protons respectively. The aromatic protons belonging to fused benzene ring was exhibited around d 6.87 to $7.37 \mathrm{ppm}$ presenting four protons. The major spectral change was observed in the IR spectrum which provides with an appearance of primary amine functional group at 3411. cm-1. In the next step, Schiff bases (3-22) were formed by refluxing 2 with various substituted aromatic aldehydes 
in the presence of few drops of glacial acetic acid. These compound were confirmed on the basis of spectral studies; ${ }^{1} \mathrm{HNMR}$ spectra showed, in each case, the signals as multiplet at $6.11-8.55 \mathrm{ppm}$ attributed to Ar-H in addition to the singlet at the $\mathrm{N}-\mathrm{H}$ group in the region 8.77-10.89 $\mathrm{ppm}$. The singlet also appeared at $\mathrm{d}$ 8.93-10.86 ppm attributed to one proton of $\mathrm{N}=\mathrm{CH}$. Thus, it confirmed the formation of Schiff bases. The final derivatives of this series $3\{[2$-chloro-3-substituted4-oxoazetidin-1-yl] imino -1, 3-dihydro-2H-indol-2-one (23-42) were synthesized by carrying out cyclization of compounds (3-22) with chloroacetyl chloride in presence of triethylamine. These products were obtained in satisfactory yield and purity as studied on the thin layer chromatography and melting point studies. The structural confirmation was carried out on the basis of spectral studies, the IR spectra of these compounds exhibited absorbance for important functional groups, such as secondary amide at $3267-3395 \mathrm{~cm}^{-1}$; the carbonyl group is indicated at $1699-1745 \mathrm{~cm}^{-1}$ and the $\mathrm{C}=\mathrm{N}$ bond is reflected around $1521-1586 \mathrm{~cm}^{-1}$. These groups are common to all the molecules from final derivatives. The ${ }^{1} \mathrm{H}$ NMR spectra of these compounds exhibited several characteristic NMR shifts. The ${ }^{1} \mathrm{H}$ NMR spectra showed, in each case the signals as multiplet at $\delta$ 7.07$8.87 \mathrm{ppm}$ attributed to $\mathrm{Ar}-\mathrm{H}$ in addition to the singlet of the $\mathrm{N}-\mathrm{H}$ group in the region $\delta 8.45-9.45 \mathrm{ppm}$. The singlet appeared for $\mathrm{C}-3$ of the azetidinone ring in the regions $\delta$ 5.07-6.88 ppm integrating for one proton. The singlet also appeared at $\delta 5.04-6.91 \mathrm{ppm}$ attributed to one proton of $\mathrm{CH}-\mathrm{Cl}$. Thus, it confirmed the formation of indole ring containing azetidinone derivatives. EI-MS of all compounds displayed the $[\mathrm{M}+\mathrm{H}]^{+}$confirming their molecular weight. The elemental (CHN) analyses were found within the limit of theoretical values. $\left(\mathrm{cm}^{-1}\right)$

\section{Pharmacological screening}

The compounds (23-42) were evaluated for antidepressant activity by forced swim test (FST) in mice at dose of $100 \mathrm{mg} / \mathrm{kg}$ and compared with the standard drug fluoxetine $(20 \mathrm{mg} / \mathrm{kg})$. There were no mortality and noticeable behavioural changes in acute oral toxicity for all the groups tested. The synthesized compounds were found to be safe upto $2000 \mathrm{mg} / \mathrm{kg}$ body weight. Initially, dose-dependent study of compound 23 at different doses $(25,50,100$, and $200 \mathrm{mg} / \mathrm{kg}$, i.p.) were performed to ensure the maximum effective dose for new synthesized compounds as antidepressant in FST. From this study, we found that $100 \mathrm{mg} / \mathrm{kg}$ is the maximum effective dose and therefore was selected for further pilot study of antidepressant-like effects of compounds (23-42) in FST. Antidepressant activity was assessed as mean immobility time in seconds, and data has been presented as Table 2 .

The standard fluoxetine reduced immobility times to $70.93 \%$ at a dose level of $20 \mathrm{mg} / \mathrm{kg}$. In our research all the synthesized derivatives can produce significant reduction in the immobility time when compared to the standard drug. Compounds $\mathbf{2 6}$ and $\mathbf{3 6}$ were found to be the most potent derivatives from the series, showing percentage decrease in immobility duration 66.82 and 65.61 respectively. At the same time, compounds 28, 38,40 and 42 showed moderate activity while compounds 25, 27 and 35 showed poor activity. Further, in order to compare the antidepressant effect of active compounds 26 and 36 with that of the standard drug, a separate study was carried out by employing doses of $20 \mathrm{mg} / \mathrm{kg}$ i.p. of 26,36 and fluoxetine, the results are presented in Figure 3.

Compounds 26 and 36 at the dose of $20 \mathrm{mg} / \mathrm{kg}$ i.p exhibited significant antidepressant activity compared with fluoxetine. The preliminary SAR of indole bearing azetidinone ring suggested that substitution of the phenyl ring by electron withdrawing groups on ortho position lead to an increase of antidepressant activity $(26,36)$. Whereas compounds containing electron releasing groups at ortho and para position of the aromatic core showed good antidepressant activity (28, 38, 40, 42). Moreover, compounds having electron releasing groups $\left(\mathrm{CH}_{3}, \mathrm{OH}\right.$, and $\left.\mathrm{OCH}_{3}\right)$ only at para position of the aromatic nucleus caused decrease in antidepressant activity $(25,27,35)$. Therefore, such compounds would represent a useful matrix for the development of a

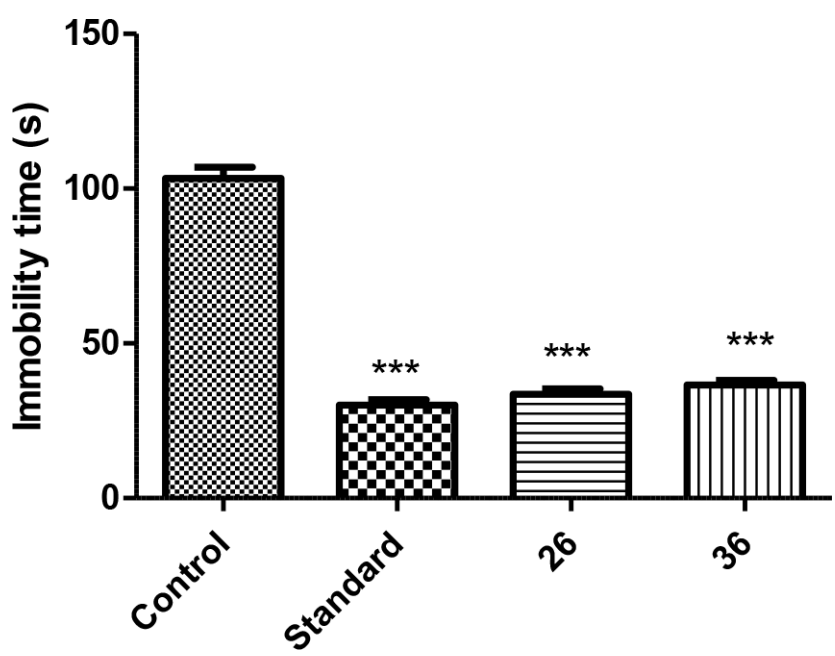

Figure 3: Effect of treatment with 26 and $36(20 \mathrm{mg} / \mathrm{kg}$, i.p.) and fluoxetine $(20 \mathrm{mg} / \mathrm{kg}$, i.p.) on the immobility time in forced swim test. (Values are represented as mean \pm S.E.M. $(n=6)$. Values are significant at ${ }^{* \star *} P<0.001$, compared with control group.). 


\begin{tabular}{|c|c|c|c|c|c|}
\hline \multicolumn{5}{|c|}{ Table 3: Molecular docking calculations of some final derivatives and standard drug. } \\
\hline Sr no. & Ligand & $\begin{array}{c}\text { Binding score } \\
\text { (Kcal/mol) }\end{array}$ & $\begin{array}{c}\text { Number of } \\
\text { hydrogen bonds }\end{array}$ & $\begin{array}{c}\text { Number of } \\
\text { hydrophobic } \\
\text { bonds }\end{array}$ & $\begin{array}{c}\text { Number of Van } \\
\text { der Walls bonds }\end{array}$ \\
\hline 1. & 26 & -2.8474 & 01 & 20 & 164 \\
\hline 2. & 28 & -2.1883 & 02 & 25 & 139 \\
\hline 3. & 36 & -2.4503 & 01 & 29 & 140 \\
\hline 4. & 38 & -2.0305 & 01 & 46 & 146 \\
\hline 5. & 40 & -0.8168 & 01 & 37 & 116 \\
\hline 6. & 42 & -0.4349 & 01 & 21 & 148 \\
\hline 7. & Fluoxetine & -3.1220 & 01 & 65 & 155 \\
\hline
\end{tabular}

new class of clinically useful antidepressant agents and deserve further investigation and derivatization.

\section{Docking study}

In order to investigate the molecular interactions and binding modes of titled derivatives, we docked these derivatives with MAO-A enzyme (PDB code 2BXS) using Biopredicta $\bigcirc$ module on the Vlife MDS Molecular Modeling software, version 4.3.1. The Vlife MDS suit uses k-nearest neighbour genetic algorithm (KNN-GA) method for molecular docking. Prior to carrying out docking the MAO-A enzyme was prepared for docking by removing the ligands and water molecules. The docking was carried out for all synthesized compounds. The molecular docking calculations including docking score and type of interaction for some derivatives and standard drug are shown in Table 3. All the synthesized derivatives possess a common binding mode and docked almost similar positions in the active site of enzyme. The docking mode of 26 and 36 with all three important interactions like hydrogen bonding hydrophobic interaction and the Van der Waals forces interactions are shown in Figure 4. It was also observed with the results of molecular docking; the energies for 26 and 36 were found to be -2.8474 and -2.4503 , respectively, which is highest in the series of synthesized derivatives and comparable to standard drug fluoxetine with score of -3.1220 .

Compound 26 revealed that it binds to the active site of MAO-A enzyme by forming hydrogen bond with GLU446A [bond length: 1.981 A; Figure 26 (a)]. Hydrophobic interactions were found to be mostly between GLY 22A, ILE 23A, SER 24A, GLY 434A, ALA 448A and FAD 600 A with bond length between 2.0 and 4.709 $\AA$ [Figure 26 (b)]. It also formed Van der Wall's interaction with enzyme amino acids having bond length between 2.0 and $3.9 \AA$ [Figure 26 (c)].

Compound 36 prominently displayed that it binds to the active site of MAO-A enzyme by forming hydrogen

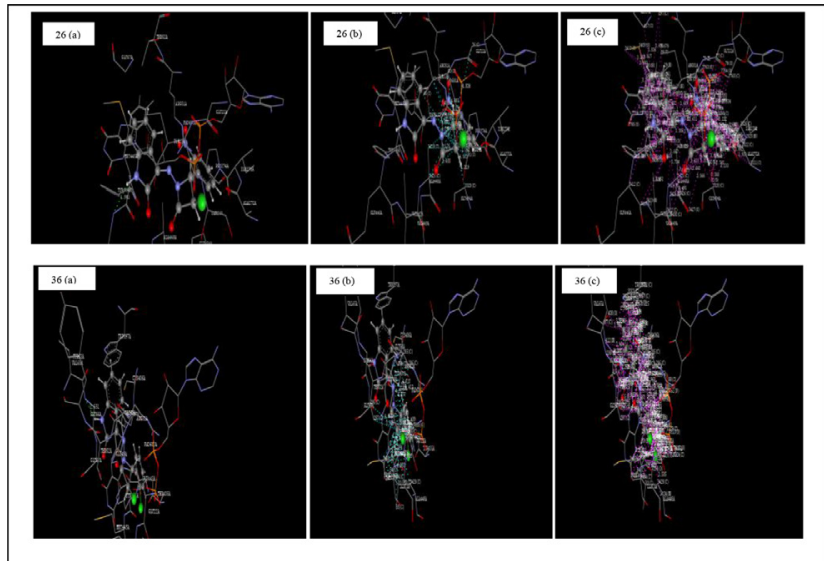

Figure 4: Molecular docking results for compound 26 (above) and 36 (below): (a) hydrogen bond interaction; (b) hydrophobic interaction; (c) Van der Waals interaction.

bond with GLY 66A [bond length: 2.551 A; Figure 36 (a)]. Hydrophobic interactions were found to be mostly among ILE 23A, ARG 51A, THR 52A, MET 445A, ALA 448A, FAD 600A with bond lengths between 4.26 and $4.93 \AA$ [Figure 36 (b)]. It also formed Van der Wall's interaction with enzyme amino acids having bond length between 2.81 and $3.85 \AA$ [Figure 36 (c)]. Apart from the hydrogen and hydrophobic bonding, a crucial $\pi-\pi$ interaction with TRP 397A with bond length 3.511. In all other synthesized derivatives ring $A$ and ring $B$ were stabilized by both hydrophobic and hydrogen bonding interaction, but ring $\mathrm{C}$ was devoid of such type of drug-receptor interaction. In the compound 28, 29, 31 and 38, the ring experiences an extra hydrophobic interaction due to the presence of a para substituted hydroxy, dimethyl amino, trimethoxy and dimethoxy group in the phenyl system respectively. Interestingly the significant steric hindrance in ring $\mathrm{C}$ can contribute an extra hydrophobic zone which resulted in computational docking scores that are in good agreement with their experimental values. Possible binding interactions of 
Extra hydrophobioc zone

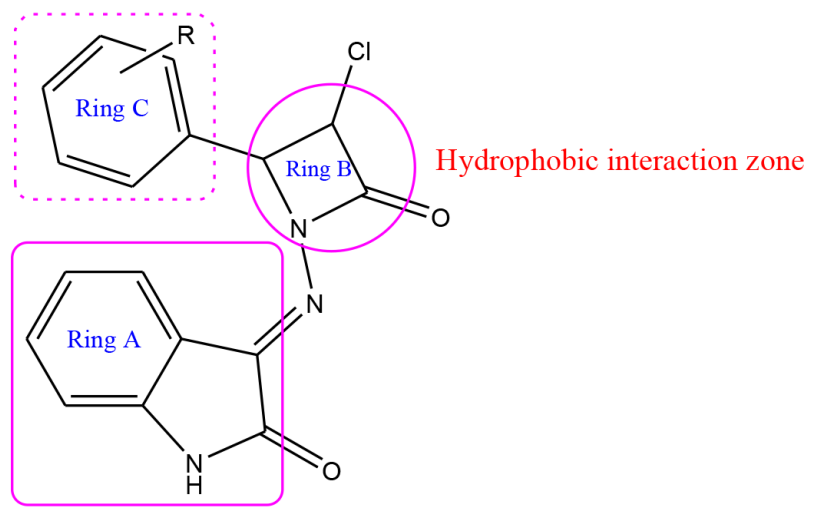

Hydrogen bonding zone

Figure 5: Binding interaction of newly designed molecule towards MAO-A.

titled scaffold obtained after the docking with enzyme residue are shown in Figure 5.

\section{CONCLUSION}

In summary, we have described the synthesis of novel indole bearing azetidinone derivatives by conventional method with high purity and better yields of product. All the spectral studies were in good agreement with the final structures of the titled derivatives. All synthesized compounds were evaluated for antidepressants activity in FST. Among all derivatives tested in the present study, compounds 26 and 36 exhibiting promising antidepressant activity comparable to that of the fluoxetine. The nitro and chloro substitution on the ortho position of phenyl ring system provided with active compounds having percentage decrease in immobility duration of 66.82 and 65.61 respectively. Molecular docking studies are also in agreement with the pharmacological evaluation with potent compounds exhibiting dock score of -2.8474 . It also suggested that the high lipophilic group in ring C can experience an extra hydrophobic binding region that can contribute a significant pharmacological activity toward the CNS depressant activity. It can be stated that these compounds can be further studied for their structure-activity relationship (SAR) studies and developed into potential lead molecules. So our research can make a great impact on those medicinal chemists who work on the development of MAO-A inhibitors.

\section{ACKNOLWEDGEMENT}

Authors are thankful to SAIF, Chandigarh, Panjab (India) for carrying out the spectral analysis of synthesized compounds.

\section{CONFLICT OF INTEREST}

Authors have no conflict of interest with the content of this article.

\section{ABBREVIATION USED}

(MAO): Monoamine oxidase; (MAO-A): Monoamine oxidase-A; (MAO-B): Monoamine oxidase-B; (CNS): Central Nervous System; (OECD): Organization for Economic Cooperation and Development; (ANOVA): Analysis Of Variance; (SEM): Standard Error Mean; (PDB): Protein Data Bank.

\section{REFERENCES}

1. Schechter LE, Ring RH, Beyer CE. Innovative approaches for the development of antidepressant drugs: current and future strategies. Neuro therapeutics. 2005;2(4):590-11.

2. Tran PV, Bymaster FP, McNamara RK, Potter WZ. Dual monoamine modulation for improved treatment of major depressive disorder. J. Clin. Psychopharmacol. 2003;23(1):78-86.

3. Tipton KF, Boyce S, O'Sullivan J, Davey GP, Healy J. Monoamine oxidases: certainties and uncertainties. Curr. Med. Chem. 2004;11(15):1965-82.

4. Edmondson DE, Mattevi A, Binda C, Li M, Hubálek F. Structure and mechanism of monoamine oxidase. Curr. Med. Chem. 2004;11(15):1983-93.

5. Rudorfer MV, Potter WZ. Antidepressants: a comparative review of the clinical pharmacology and therapeutic use of the "newer" verses the "older" drugs. Drugs. 1989;37:713-38.

6. Wouters J. Structural aspects of monoamine oxidase and its reversible inhibition. Curr. Med. Chem. 1998;5(2):137-62.

7. Manna K, Aggarwal Y. Microwave assisted synthesis of new indophenazine 1, 3, 5-trisubstituted pyrrazoline derivatives of benzofuran and their antimicrobial activity. Bioorg. Med. Chem. Lett. 2009;19(10):2688-92.

8. Jordan MA, Wilson L. Microtubules as a target for anticancer drugs. Nat. Rev. Cancer. 2004;4: 253-65.

9. Shibinskya MO, Lyakhov SA, Mazepa AV, Andronati SA. Synthesis, cytotoxicity, antiviral activity and interferon inducing ability of 6-(2-aminomethyl)-6H-indolo [2, 3-b] quinoxalines. Eur. J. Med. Chem. 2010;45(3):1237-1243.

10. Palluotto F, Carotti A, Casini G, Camagna F, Genchi G, Structure activity relationship of 2-aryl-2, 5 dihydropyridazino [4,3-b] indol-3-(3H)-ones at the benzodiazepine receptor. Bioorg. Med. Chem. 1996;4(12):2091-04.

11. Kerzare DR, Khedekar PB. Indole Derivatives acting on Central Nervous System - Review. J Pharm Sci Bioscientific Res. 2016;6(1):144-56.

12. Trilok C, Neha G, Ashok K. Synthesis and Anti-inflammatory Activity of Indole Derivative. International Journal of Chem Tech Research. 2010;2(2):762-73

13. Desh D, Anjali T, Santosh KS. Synthesis, antimicrobial and antitubercular screening of new azetidinone derivatives. J. chem. pharm. res. 2013;5(10):134-40.

14. Kumar R, Shukla A, Tyagi DS. An efficient synthesis of bio-active azetidinones and thiazolidinones of 3-methyl-1-phenyl-1H-Pyarazol-5-ol. Int. J. Sci. Res. 2012;2(6):1-7.

15. Patel V, Bhatt ND, Patel PM, Bhatt $P$, Joshi HD. Synthesis and characterization of some 5H-dibenzo (b,f)azepine-5- carboxamido-4'-aryl-3'-aryl piperazine2'-azetidinone derivatives. Der Chemica Sinica. 2012;3(2):359-63.

16. Sugumaran M, Sethuvani S, Poornima M. Synthesis, characterization and antimicrobial evaluation of 2-azetidinone and 4-thiazolidinone derivatives. Res. J. Pharma. Bio. Chem. Sci. 2012;3(2):625-31.

17. Samadhiya P, Sharma R, Srivastava SK, Srivastava SD. Synthesis of 2-azetidinone derivatives of 6 -nitro- $1 \mathrm{H}$-indazole and their biological importance. Quim. Nova. 2012;35(5):914-19.

18. Traynor K. New drug and biological product approvals, 2011 Am J Health Syst Pharm. 2012;69:180-82. 
19. Ergenec N, Gunay NS, Damirdamar S. Synthesis and antidepressant evaluation of new 3-phenyl-5-sulfonamidoindole derivatives. Eur. J. Med. Chem. 1998;33(2):143-48.

20. Shelke SM, Bhosale SH. Synthesis, antidepressant evaluation and QSAR studies of novel 2-(5H-[1,2, 4]triazino[5,6-b]indol-3-ylthio)-N-(substituted phenyl) acetamides. Bioorg. Med. Chem. Lett. 2010;20(15):4661-64.
21. Porsolt RD, Anton G, Blanet N, Jalbre M. Behavioural despair in rats: a new model sensitive to antidepressant treatments. Eur. J. Pharm. 1978;47:379-86.

22. OECD, 2001-gudeline on acute oral toxicity (AOT) Environmental health and safety monograph series on testing and adjustment No. 425 .

\section{PICTORIAL ABSTRACT}

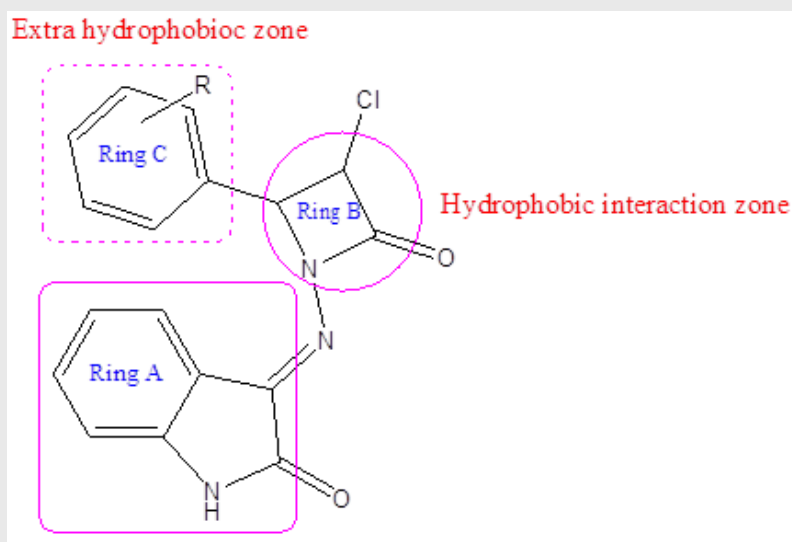

Hydrogen bonding zone

\section{SUMMARY}

- A novel series of indole bearing azetidinone derivatives were synthesized, characterized and evaluated for antidepressant activity along with molecular docking studies. The research aimed at generation of new molecular template by linking two pharmacophores (Indole and Azetidinone). From the series of twenty compounds only two compounds 26 and 36 demonstrated as promising molecules with percentage decrease in immobility duration 66.82 and 65.61 respectively. These compounds could therefore may have enough potential to be evaluated as antidepressant agents and can make a great impact on those medicinal chemists who work on development of MAO-A inhibitors.

\section{About Authors}

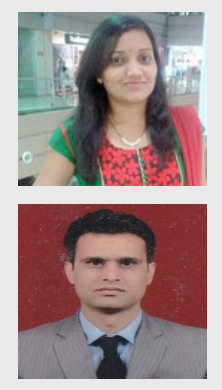

Deweshri R. Kerzare: Is currently teaching medicinal and computational chemistry at both undergraduate and postgraduate levels. Her current area of interest involves computer assisted rational designing and synthesis of various indole derivatives. She has published few research, review and book chapter in journals of international repute.

Sunil S. Menghani: Is presently working as Assistant Professor in Department of Pharmaceutical Chemistry, Yenepoya Pharmacy College and Research Centre, Magluru Karnataka. He has more than 9 years of teaching medicinal and organic chemistry at both undergraduate and postgraduate levels. His research interest includes rational design of small molecules of benzodiazepines which are acting on CNS.

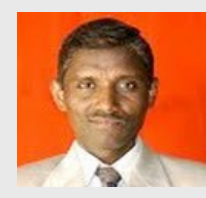

Pramod B. Khedekar: Is presently working as Professor at Department of Pharmaceutical Sciences, RTMNU, Nagpur. He is the man of substance, progress personified, visionary and an institution in himself. He supervised $10 \mathrm{Ph}$.D. and $42 \mathrm{M}$.Pharm. students. He worked for the improvement of curricula in various academic bodies and introduced various graduate and post-graduate courses in chemistry. He is a member of a number of professional and scientific societies as well as serving the position of reviewer and editorial board member of various international journals.

Cite this article: Kerzare DR, Menghani SS, Khedekar PB. Synthesis, Characterization, Antidepressant Activity and Docking Studies of Some Novel Indole Bearing Azetidinone Derivatives. Indian J of Pharmaceutical Education and Research. 2018;52(1):110-21. 